\title{
Assessment of myocardial viability
}

\section{Vitalitätsdliagnostilk nach myokardialer Ischämie}

Therapieansätze, deren Ziel eine möglichst frühzeitige und vollständige Reperfusion des Infarktareals ist, wie die Notfallherzkatheteruntersuchung mit Infarktgefäßrekanalisation oder bei fehlender frühzeitiger Interventionsmöglichkeit die Thrombolyse, haben zusammen mit sekundärpräventiven Maßnahmen in den letzten Jahren zu einer deutlichen Reduktion der kardialen Mortalität geführt. Dennoch bleibt der postinfarzielle Verlust kontraktilen Myokards die wichtigste Ursache für die Entwicklung einer Linksherzinsuffizienz. Da der Verlust kontraktilen Myokards nur begrenzt medikamentös kompensiert werden kann, hat sich das klinische Interesse vermehrt auf die Erfassung postinfarziell kontraktionsgestörter, aber noch vitaler, das heißt potenziell erholungsfähiger Myokardregionen fokussiert. Mit Hilfe moderner bildgebender Verfahren wie der Stressechokardiographie, der Magnetresonanztomographie und $\rightarrow$ szintigraphischer Schnittbilduntersuchungen ist es heute möglich, irreversibel geschädigtes Narbengewebe von kontraktionsgestörtem, aber noch vitalem Myokard zu unterscheiden. Gelingt der Nachweis vitalen Myokards, können revaskularisierende Maßnahmen mit Herzkathetertechniken oder Bypassoperation zu einer Erholung der Kontraktionsfunktion und damit einer Verbesserung der Prognose führen (1). Wird dagegen postinfarziell vitales Myokard nicht revaskularisiert, ist die Prognose für solche Patienten sogar schlechter als für diejenigen ohne Vitalitätsnachweis, da diese vitalen, aber minderperfundierten Regionen wahrscheinlich bei der Induktion maligner Herzrhythmusstörungen eine Rolle spielen. Aber auch bei Patienten mit chronischer koronarer Herzerkrankung, von denen einige bei Diagnosestellung noch keinen Herzinfarkt erlitten haben, oder bei denen ein klinisch inapparent verlaufener Myokardinfarkt evtl. bereits einige Jahre zurück liegt, ist die Vitalitätsdiagnostik prognostisch wichtig und damit auch indiziert (Abb.1) $(\rightarrow$ Weitere Informationen zur myokardialen Minderperfusion). Lässt sich dagegen kein vitales Myokard nachweisen, bleibt dem Patienten nach einem Herzinfarkt eine von vorneherein aussichtslose und zudem mit Risiken belastete revaskularisierende Therapie zugunsten einer primär medikamentösen Therapie oder einer Herztransplantation erspart.

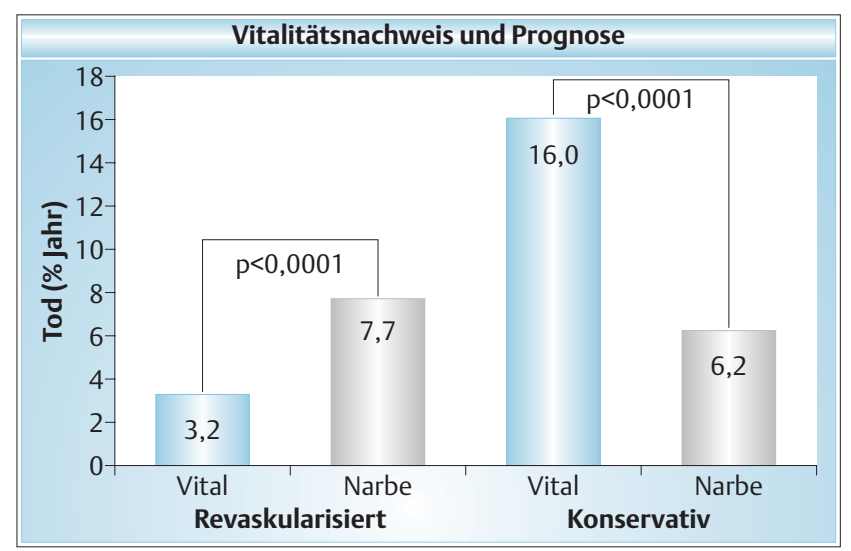

Abb.1 Vitalitätsnachweis und Prognose. Von 3088 Patienten mit einer mittleren LVEF (=linksventrikuläre Ejektionsfraktion) von $32 \%$ wurde bei $35 \%$ eine Revaskularisierung und bei $65 \%$ eine konservative Therapie durchgeführt. In der Gruppe der Patienten mit Revaskularisierung war die Sterblichkeit in einem mittleren Beobachtungszeitraum von 25 Monaten mit 3,2\% vs. 7,7\% signifikant niedriger. Im Gegensatz dazu war die Sterblichkeit der Patienten mit konservativer Behandlung ohne Nachweis von vitalem Myokard mit 16\% vs. 6,2\% signifikant höher im Vergleich zu Patienten ohne Nachweis von vitalem Myokard. Die Studie impliziert, dass eine nicht durchgeführte oder technisch nicht mögliche Revaskularisierung bei Nachweis von vitalem Myokard die Prognose der betroffenen Patienten verschlechtert (nach (1)).

kurzgefasst: Myokardiale Vitalitätsdiagnostik soll Patienten mit linksventrikulären Kontraktionsstörungen identifizieren, bei denen durch eine Myokardrevaskularisation 1. eine Verbesserung der regionalen Kontraktionsfunktion und 2. eine Verbesserung der Prognose erreicht werden kann.

1 Mit „ $\rightarrow$ “ sind Textstellen gekennzeichnet, zu denen Sie zusätzliche Texte und Bilder im Internet unter www.thieme.de/dmw/cme finden können. 


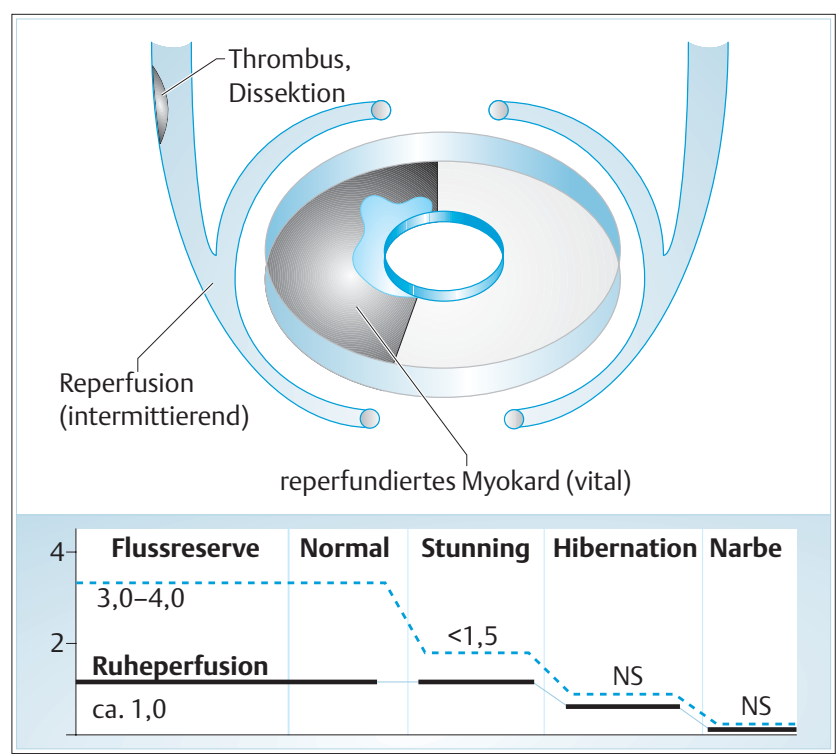

Abb.2 Kontraktile Dysfunktion - Stunning. Definition: Reperfusion nach einmaliger oder repetitiver Ischämiephase mit vorübergehender Kontraktionsstörung und spontaner Erholung der kontraktilen Funktion. Die koronare Flussreserve ( $\mathrm{ml} / \mathrm{g}$ Herzgewicht/min) ist deutlich reduziert bei (intermittierend) normaler Ruheperfusion (NS=nicht stimulierbar).

\section{Pathophysiologische Aspelkte der myolkardlialen Vitalität}

\section{Stunning}

Die postischämische, teilweise erhebliche Funktionsstörung des noch nicht irreversibel geschädigten Myokards (z.B. nach erfolgreicher Thrombolyse-Behandlung) wird als myokardialer Schockzustand oder „Stunning“ bezeichnet (Abb.2). Unter „Stunning“ wird eine reversible postischämische linksventrikuläre Funktionsstörung nach einer länger andauernden akuten oder repetitiven Ischämiephase verstanden (4). Die regionale Funktionsstörung wird hervorgerufen durch den kombinierten Effekt von Ischämie und Reperfusionsschaden. Das klinische Korrelat für Stunning kann z.B. der akute Myokardinfarkt nach interventionell vermittelter oder spontaner Reperfusion oder das akute Koronarsyndrom mit intermittierender thrombotischer Verlegung des Gefäßlumens sein.

\section{Hibernation}

Im Gegensatz zum „Stunning“ findet sich beim winterschlafenden oder „Hibernating“ Myokard (Abb.3) eine chronische Minderdurchblutung der Infarktzone ( $<1 \mathrm{ml} / \mathrm{g}$ Herzgewicht/min) bei aufgehobener koronarer Flussreserve (12). Um unter diesen ungünstigen Perfusionsbedingungen einen schweren Sauerstoffmangel zu vermeiden wird nach einer klinischen Modellvorstellung die Pumpfunktion der betroffenen Herzmuskelbereiche gleichsam eingefroren damit die Restperfusion des Herzmuskels ausreicht um den Ruhestoffwechsel aufrechtzuerhalten. Dieser Zustand ist aber nicht stabil. Während ein weiteres Absinken der Restperfusion zu einer progressiven Dedifferenzierung und ultrastrukturellen Schädigung des Myokards bis hin zur Nekrose führt, kann eine Verbesserung der Perfusion durch revaskularisierende Maßnahmen eine in Abhängigkeit von der Vorschädigung (Fibrose, subendokardialer Infarkt) mehr oder weniger vollständige Wiederherstellung der kontraktilen Funktion bewirken. Das klinische Korrelat der regionalen

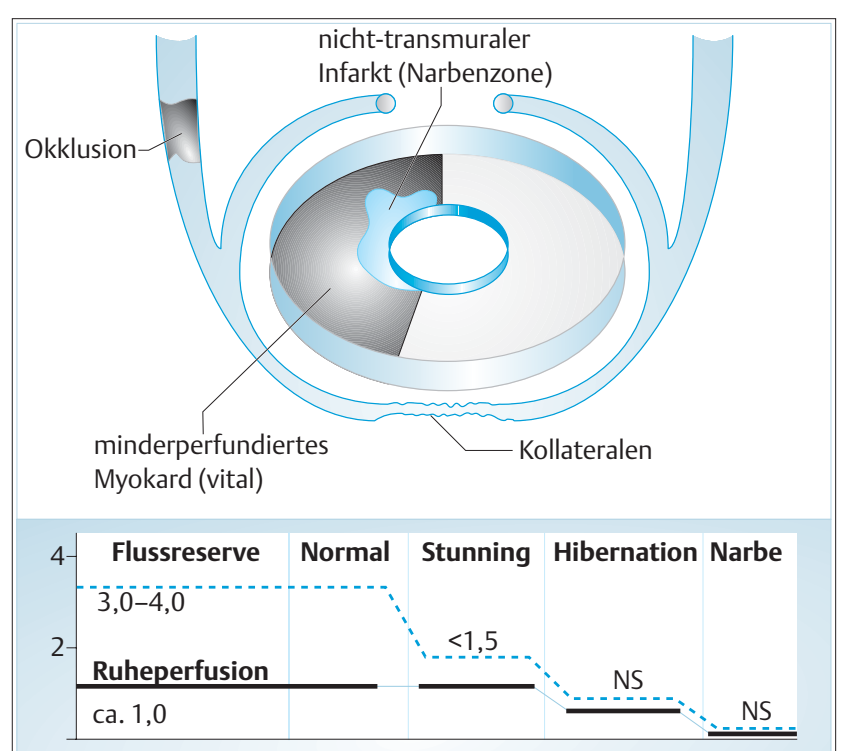

Abb.3 Kontraktile Dysfunktion - Hibernation. Definition: Chronische Minderperfusion (fehlende koronare Flussreserve in $\mathrm{ml} / \mathrm{g}$ Herzgewicht/ min) einhergehend mit strukturellem Umbau des Myokards (Fibrose) und persistierender Kontraktionsstörung. Eine Erholung der kontraktilen Funktion ist nur nach erfolgreicher Revaskularisierung in Abhängigkeit vom Ausmaß der schon bestehenden Myokardfibrose möglich (NS=nicht stimulierbar).

oder globalen Dysfunktion bei winterschlafendem (hibernating) Myokard ist die hochgradige Koronarstenose oder der vollständige Verschluss einer Koronararterie mit Kollateralversorgung der abhängigen Myokardregion. Möglicherweise führen auch repetitive Ischämiephasen (repetitives Stunning), wie z.B. Belastungssituationen bei Koronararterienverschluss mit nicht ausreichender Kollateralversorgung zu winterschlafendem Myokard.

\section{Klinik und EKG}

Nach einem Infarkt gibt es bei einem Teil der Patienten klinische (Angina pectoris) und elektrokardiographische (fehlende Q-Zacken) Anhaltspunkte für das Vorhandensein von vitalem Myokard. Häufig gelingt es aber anhand klinischer Parameter und EKG nicht, zwischen vitalem und irreversibel geschädigtem Myokard zu unterscheiden. Obwohl die Koronarangiographie entscheidend ist für die Absicherung der Diagnose KHK und letztlich für die Abklärung, ob und in welcher Form eine Revaskularisierung möglich ist, erlaubt auch sie keine sichere Differenzierung zwischen vitalen Myokardarealen und irreversibel geschädigtem Narbengewebe. Besteht koronarmorphologisch die Möglichkeit zur Revaskularisierung, sollte deshalb durch eine weiterführende bildgebende Diagnostik der Vitalitätsstatus kontraktionsgestörter Myokardregionen überprüft und darauf basierend über eine weitere medikamentös-konservative oder revaskularisierende Therapie entschieden werden. Die Differenzierung zwischen vitalem Myokard und Narbengewebe gelingt durch eine Reihe von Parametern, die auf der Erfassung von Morphologie, Funktion, Perfusion, Integrität der Zellmembran und dem Zellstoffwechsel beruhen. Die Bandbreite dieser Vitalitätsparameter kann nicht durch eine einzige Untersuchungstechnik abgedeckt werden, sondern einzelne Untersuchungstechniken erfassen jeweils mehr oder weniger große und klinisch unterschiedlich bedeutsame Teilbereiche dieses Spektrums (Tab. 1). 


\section{Echokardiographie}

\section{Ein- und zweidimensionale Echokardiographie}

Die Echokardiographie in Ruhe ist die wegweisende Untersuchung zur Erfassung der Ausdehnung einer postinfarziellen Wandbewegungsstörung und des daraus resultierenden Grades der linksventrikulären Pumpfunktionsstörung. Bei ausgeprägter Wandverdünnung in dyskinetischen Myokardregionen (Aneurysmata) ist die Wahrscheinlichkeit für das überwiegende Vorliegen von irreversibel geschädigtem Narbengewebe sehr groß. A- oder dyskinetische Areale ohne Wandverdünnung können avital im Frühstadium der Narbenbildung, oder aber auch nicht-transmurale Infarktzonen mit Restvitalität sein. Andererseits deutet eine Hypokinesie per se auf einen gewissen Grad an Restvitalität hin. Bei serieller Untersuchung (zunächst akute Infarktphase, dann vor Krankenhausentlassung) weist eine Funktionsverbesserung oder -normalisierung im infarzierten Areal auf eine erhalten gebliebene Vitalität nach reversibler Ischämie (=Stunning) hin. Besonderes Augenmerk hinsichtlich ihrer Prognose verdienen Patienten mit höhergradiger Einschränkung der linksventrikulären Funktion ( $\mathrm{EF}<40 \%$ ).

kurzgefasst: Ein echokardiographisch a- oder dyskinetisches Myokardareal in Ruhe kann sowohl avital als auch vital sein, bzw. Anteile vitaler und avitaler Bereiche enthalten. Bei serieller Durchführung kann die postischämische Wiederherstellung der Funktion im Infarktareal nach erfolgreicher Therapie dokumentiert werden („Stunned myocardium“). Postischämisch persistierende Wandbewegungsstörungen (V.a. „Hibernating Myocardium") erfordern eine weiterführende Vitalitätsdiagnostik mit der Belastungsechokardiographie.

\section{Dobutamin-Stressechokardiographie}

Die durch Studiendaten am besten evaluierte echokardiographische Methode zur Vitalitätsdiagnostik ist die Dobutamin-Stressechokardiographie. Der Vorteil der pharmakologischen Belastung im Vergleich mit der dynamischen Fahrradergometrie besteht in der einfacheren Durchführbarkeit (= Darstellbarkeit) bei optimierter Seitenlagerung des ruhenden Patienten. Das Titrationsprotokoll sieht 3-minütige Phasen von 5, 10, 20, 30, 40 $\mu \mathrm{g} / \mathrm{kg} / \mathrm{min}$ kontinuierlich über einen Perfusor intravenös gegebenem Dobutamin vor. Bei Nichterreichen der submaximalen Ausbelastungsfrequenz ([220-Lebensalter]×0,85) erfolgt in 1-minütigen Abständen die fraktionierte Atropingabe von jeweils $0,25 \mathrm{mg}$. Abbruchkriterien sind das Erreichen der submaximalen Ausbelastungsfrequenz und/oder der Nachweis einer belastungsinduzierten Ischämiereaktion. Eine Notfallausrüstung einschließlich Defibrillator muss hierfür vor Ort verfügbar sein.

Ziel der stufenweise Dobutaminbelastung ist das „Aufwecken“ winterschlafenden (= hibernierenden) oder des gelähmten (= stunning) Myokards (Kontraktionsreserve), das unter Ruhebedingungen als akinetisch oder dyskinetisch beurteilt wurde. In niedriger Dosis (5$10 \mu \mathrm{g} / \mathrm{kg} / \mathrm{min}$ ) führt die $\beta_{1}$-adrenerge Stimulation zur Rekrutierung der Restkontraktilität im postischämisch kontraktionsgestörten Myokardareal. Dabei kommt es zur Wanddickenzunahme und endokardialen Einwärtsbewegung. Liegt gelähmtes (=stunning) Myokard vor, so bleibt diese „Erweckung zur Funktion“ bis zur Zieldosis der Stressechokardiographie-Protokolls $(40 \mu \mathrm{g} / \mathrm{kg} / \mathrm{min})$ erhalten (monophasische Antwort). Liegt dagegen winterschlafendes

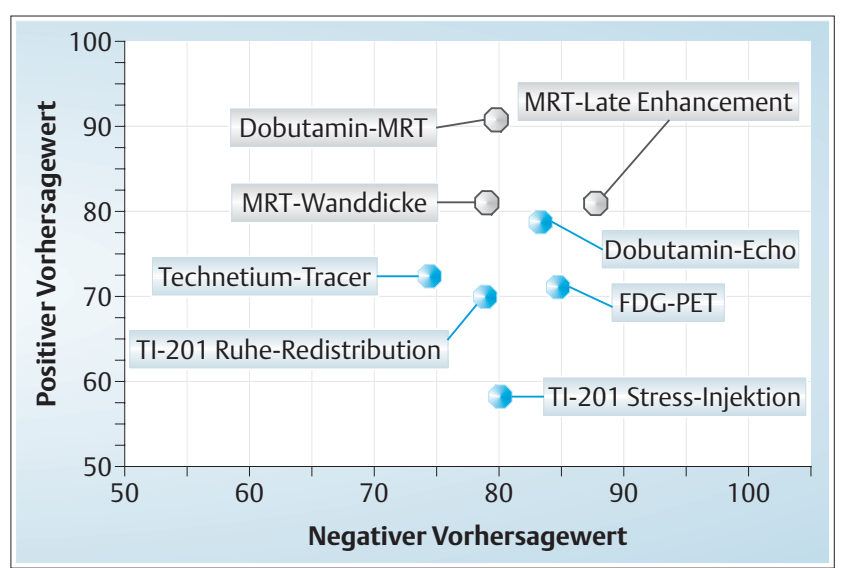

Abb.5 Methodenvergleich: Funktionelle Erholung. Die Datenlage für die dargestellten Untersuchungstechniken ist sehr unterschiedlich. Die guten Ergebnisse der noch jungen MRT-Techniken (grau) werden durch die noch geringe Datenbasis relativiert, während für alle anderen Techniken (blau) eine fundierte Datenbasis vorliegt.

( = hibernierendes) chronisch ischämisches Myokard vor, so kann bei niedriger Dobutaminbelastung $(5-10 \mu \mathrm{g} / \mathrm{kg} / \mathrm{min})$ zunächst eine Kontraktionsreserve nachgewiesen werden, bei höherer Dobutamin-Dosierung dominiert jedoch die belastungsinduzierte Ischämie, die durch einen erneuten Funktionsverlust im Infarktareal gekennzeichnet ist. Diese dadurch entstehende „biphasische“ Antwort ist typisch für „hibernation“ $\rightarrow$ (Abb.4). Die kumulative Sensitivität/ Spezifität der Methode hinsichtlich der funktionellen Erholung nach Revaskularisierung liegt bei Zusammenschau von 19 Studien bei $81 \%$ bzw. $86 \%$, der positiv prädiktive Wert bei $83 \%$ (14). Da bekannt ist, dass sich „Stunned myocardium“ innerhalb weniger Tage (maximal 2 Wochen) nach Infarkt spontan erholt, sollte die Dobutamin-Belastungsechokardiographie zur Erkennung hibernierenden Myokards frühestens 2 Wochen nach Infarkt erfolgen. Andererseits sollte die Vitalitätsdiagnostik nicht länger als 6 Wochen nach Infarkt stattfinden, da es in Arealen mit „Hibernating myocardium“ zu einem progredienten Verlust der funktionellen Reversibilität kommen kann.

kurzgefasst: Die Dobutamin-Belastungsechokardiographie identifiziert durch $\beta_{1}$-adrenerge positiv inotrope Stimulation kontraktionsgestörtes Myokard mit positiver Inotropiereserve, das auf dem Boden einer nach Infarkttherapie verbliebenen Koronarstenosierung hinsichtlich einer Funktionsverbesserung von einer Revaskularisierung profitieren wird (Hibernating myocardium). Hierzu sollte das gesamte Titrationsprotokoll eingehalten werden, um Aussagen zu Vitalität und Ischämie (biphasische Antwort) treffen zu können. Als Zeitpunkt der Diagnostik empfiehlt sich ein Zeitraum zwischen der 3. und 4. Woche nach Infarkt.

\section{Echokardiographische Vitalitätsdiagnostik: neue Methoden}

Wissenschaftlich interessant aber bisher weniger gut durch Studien belegt sind eine Reihe von neueren Belastungsprotokollen und Untersuchungstechniken. Die $\rightarrow$ dynamische FahrradergometerStressechokardiographie kann bei gut schallbaren Patienten auf einer seitlich kippbaren Ergometerliege durchgeführt werden. Die Patienten werden zunächst für 3 Minuten mit 25 Watt belastet (6). Dies entspricht in etwa einer low-dose Dobutamin-Echokardiographie. 
Bei unbekanntem Koronarstatus sollte in Analogie zur DobutaminStressechokardiographie unmittelbar eine konventionelle Stressechokardiographie mit stufenweiser Belastung bis zur submaximalen Ausbelastungsfrequenz folgen. Möglich ist auch eine $\rightarrow$ transösophageale Echokardiographie unter Dobutaminbelastung. Sie kann bei Patienten zum Einsatz kommen, die von transthorakal trotz Einsatz moderner Bildgebungsverfahren (Tissue harmonic imaging) nicht ausreichend gut schallbar sind. Vorteil ist die hervorragende Bildqualität, Nachteil der semi-invasive Untersuchungszugang (2). Einige Arbeiten existieren zur $\rightarrow$ Dipyridamol-, zur $\rightarrow$ Nitrat- und zur Enoximon-Belastungsechokardiographie. Insgesamt ergaben sich jedoch keine entscheidenden Vorteile gegenüber der Dobutamin-Belastungsechokardiographie. Zukunftsweisend in der echokardiographischen Vitalitätsdiagnostik sind neuere Arbeiten zur $\rightarrow$ Gewebe-Dopplerechokardiographie und zur $\rightarrow$ Myokard-Kontrastechokardiographie. Möglicherweise ist durch Anwendung dieser innovativen echokardiographischen Methoden in Zukunft der Einsatz eines pharmakologischen „Stressors“ nicht mehr notwendig.

kurzgefasst: Von den Fachgesellschaften empfohlen wird zur echokardiographischen Vitalitätsdiagnostik nach Myokardinfarkt die Dobutamin-Belastungsechokardiographie. Alternative pharmakologische Belastungsprotokolle existieren, basieren jedoch auf einer spärlichen Datenlage. An neueren Untersuchungsmethoden scheinen vor allem die Gewebe-Dopplerechokardiographie und die MyokardKontrastechokardiographie erfolgversprechend zu sein.

\section{Magnetresonamztomographie}

Die diagnostische Zuverlässigkeit der Stress-Magnetresonanztomographie (Stress-MRT) ist sowohl in der Ischämie- als auch in der Vitalitätsdiagnostik im Vergleich zu etablierten Verfahren (PET, Myokardszinitigraphie, Echokardiographie) hinreichend belegt. In einem direkten Vergleich zwischen Dobutamin-Stress-MRT und Dobutamin-Stressechokardiographie wurden identische Hochdosisprotokolle verwendet und die Ergebnisse beider Untersuchungstechniken bezüglich Sensitivität und Spezifität für die Erfassung bedeutsamer Koronarstenosen mit den angiographischen Daten verglichen. Dabei schnitt die MRT gegenüber der Echokardiographie hinsichtlich Sensitivität (86/74\%), Spezifität (86/70\%) und diagnostischer Zuverlässigkeit (86/73\%) signifikant besser ab. Außerdem zeigten weitere Stress-MRT-Studien, dass bei einem Großteil der Patienten mit schlechter echokardiographischer Bildqualität eine zuverlässige Ischämiediagnostik auf der Basis ischämieinduzierter Wandbewegungsstörung mittels der Stress-MRT durchgeführt werden kann.

Prinzipiell macht sich die Magnetresonanztomographie (MRT) bei der $\rightarrow$ MRT-Stressuntersuchung wie die Echokardiographie die Erfassung einer dobutamininduzierbaren Kontraktionsreserve als Vitalitätsparameter zunutze (10). Darüber hinaus kann aufgrund der hervorragenden räumlichen Auflösung auch die myokardiale Wanddicke in Systole und Diastole gemessen und daraus die Wanddickenzunahme quantitativ ermittelt werden (3). Aktuelle Weiterentwicklungen der MRT wie die ultraschnelle Bildakquisition mit der Möglichkeit einer Echtzeit-Darstellung des myokardialen Kontraktionszyklus und verbesserte Auswerteprogramme ermöglichen heute die Durchführung der MRT-Vitalitätsdiagnostik in einer der Echokardiographie vergleichbaren Untersuchungszeit von ca. 30 Minuten. Basierend auf der dobutamininduzierbaren Kontraktionsreserve ergibt sich für die MRT ein positiv prädiktiver Wert für die Erholung der linksventrikulären Kontraktionsfunktion von $87 \%$ und ein negativ prädiktiver von Wert 91\% (3). Darüber hinaus zeigen aktuelle MRT-Studien die Möglichkeit einer exakten Differenzierung zwischen vitalem Myokard und irreversibel geschädigtem Narbengewebe anhand einer verzögert auftretenden aber persistierenden Anreicherung von MRT-Kontrastmittel (Gadolinium) in der Infarktzone, die dort zu einer Signalanhebung führt (late enhancement) (7,9). Eine fehlende Kontrastmittelanreicherung (kein late enhancement) in einer akinetischen Myokardregion ist ein Indikator für vitales Myokard, während eine ausgeprägte Kontrastmittelanreicherung (late enhancement), die $>50 \%$ der Wanddicke umfasst, auf überwiegend nekrotisches Myokard hinweist ( $\rightarrow$ MRT-Infarktdiagnostik). Diese technisch im Vergleich zu den Dobutaminbelastungsuntersuchungen wenig aufwändige MRT-Kontrast-Untersuchung hat in Kombination mit der Erfassung der Myokardfunktion unter Ruhebedingungen (Wandbewegung, Wanddickenzunahme) das Potential, sich zukünftig als diagnostisch führende Technik zur Vitalitätserfassung zu etablieren $(8,18)$.

kurzgefasst: Bei der „Low-dose Dobutamin-MRT“ erfolgt eine Infusion von 5 und $10 \mu \mathrm{g} / \mathrm{kg} / \mathrm{min}$ Dobutamin für jeweils $3 \mathrm{Mi}$ nuten zur Bestimmung der Kontraktionsreserve. Bei der Kontrast-MRT wird das (transmurale) Ausmaß der irrevesibel narbigen Veränderungen in der akinetischen Infarktzone anhand der Kontrastmittelanreicherung (Gadolinium) im Infarktareal und der damit verbundenen Signalanhebung („late enhancement“) beurteilt. Ausschlusskriterien für die MRT-Vitalitätsdiagnostik sind Schrittmacher, Klaustrophobie, bestimmte Metallimplantate und Splitter.

\section{Nulklearmedizinische Verfahren}

Single Photonen Emissions Computer Tomographie (SPECT)

Zu den klinisch etablierten Techniken in der Vitalitätsdiagnostik gehört die $\rightarrow$ Myokardszintigraphie in SPECT-Technik mit Tracern wie Thallium-201 oder Technetium-Methoxyisobutylisonitril (TcMIBI) (17). Um die Sensitivität der Thallium-201-Szintigraphie zum Nachweis von vitalem Gewebe in der Infarktregion zu erhöhen, wurden neue Konzepte, wie z.B. die Thallium-201-Reinjektion oder die Ruheinjektion mit Spätaufnahmen, entwickelt. Das chronisch ischämische Myokard im Infarktareal bekommt mit dem erneuten Angebot an Thallium-201 die Möglichkeit, den Tracer unter Ruhebedingungen aufzunehmen, und vitales Gewebe kann im Rahmen einer Spätaufnahme durch eine vermehrte Traceraufnahme identifiziert werden. Hier liegt die Auffassung zugrunde, dass die verzögerte Aufnahme von Thallium-201, das einen aktiven Transportprozess erfordert, zwar durch vitale, nicht jedoch durch nekrotische Zellen erfolgen kann. Damit ist eine klare Unterscheidungsmöglichkeit zwischen vitalem Myokard und Narbengewebe basierend auf der beobachteten Thallium-201-Aufnahme gegeben. Eine Metaanalyse bezogen auf sechs Studien unter Verwendung der Thallium-201Reinjektionstechnik bei 113 Patienten mit Myokardinfarkt zeigte, dass der positiv prädiktive Wert der Thalliumszintigraphie für die Erholung der linksventrikulären Funktion nach Revaskularisierung im Mittel bei 75\% und der negativ prädiktive Wert, d.h. die korrekte Erfassung irreversibel geschädigten Myokards 74\% beträgt (15). 


\section{Positronenemissionstomographie (PET)}

Unter dem Aspekt der Stoffwechselanpassung an eine chronische Minderdurchblutung des Herzmuskels hat die Positronenemissionstomographie (PET) unter Verwendung des Tracers 18F-Fluorodeoxyglukose (FDG) sehr viel zum Verständnis der Ischämiereaktion des Myokards beigetragen. Mit der Umstellung auf den anaeroben Stoffwechsel unter chronischen Ischämiebedingungen kann die bevorzugte Aufnahme von Glukose in die Myokardzellen anstelle von Fettsäuren diagnostisch genutzt werden. Nur vitale Myokardzellen können auf die anaerobe Glykolyse umstellen und damit bevorzugt FDG aufnehmen. Dies gilt als sensibler metabolischer Nachweis für erhaltene Myokardvitalität. Aufwendiger ist die gleichzeitige Messung der myokardialen Perfusion der myokardialen Perfusion (z. B. mit Perfusionsmarkern wie N-13 Ammoniak) und der FDG-Aufnahme mit der PET. Auf der Basis dieser kombinierten Untersuchungen konnten unterschiedliche Verteilungsmuster zwischen Glukosestoffwechsel und myokardialer Perfusion definiert werden:

1. normale Perfusion und normaler Glukosestoffwechsel,

2. reduzierte Perfusion und normaler oder erhöhter Glukosestoffwechsel und

3. reduzierte Perfusion und reduzierter Glukosestoffwechsel.

Das spezifische Verteilungsmuster für vitales Myokard ist gekennzeichnet durch eine reduzierte Perfusion bei normalem oder deutlich erhöhtem Glukosestoffwechsel (Mismatch zwischen Metabolismus und Perfusion). Das typische Verteilungsmuster für Narbengewebe ist eine reduzierte oder fehlende Perfusion bei gleichzeitig reduziertem oder fehlendem Glukosestoffwechsel (Match zwischen Metabolismus und Perfusion). Der positiv prädiktive Wert einer PET-Untersuchung von Perfusion und Glukosestoffwechsel für die Erholung der linksventrikulären Funktion nach Revaskularisierung ( $\rightarrow$ weitere Informationen zu Revaskularisierung und PET) variiert in verschiedenen Studien zwischen 68\% und 95\% (im Mittel $81 \%$ ), der negativ prädiktive Wert zwischen $75 \%$ und $100 \%$ (im Mittel 84\%) (16).

\section{kurzgefasst: Die SPECT-Untersuchung mit Thallium-201 oder technetiummarkierten Tracern ist ein auf breiter Datenbasis beruhendes etabliertes Verfahren zur Vitalitätserfassung. Die PET ist ein vorwiegend wissenschaftlich eingesetzter Re- ferenzstandard der Vitalitätsdiagnostik mit der Möglichkeit zur quantitativen Perfusionsmessung (z.B. mit N-13 Ammo- niak) und Bestimmung des Myokardmetabolismus (FDG).}

\section{Klinische Bedeutung der Vitalitätsdiagnostik}

Der Nachweis vitaler Anteile in kontraktionsgestörtem Herzmuskelgewebe ist ein wichtiger Faktor im therapeutischen Entscheidungsprozess bei Patienten mit KHK, insbesondere bei Vorliegen einer schweren linksventrikulärer Funktionsstörung mit drohender Linksherzinsuffizienz. Bei diesen Patienten muss bei jeder Indikationsstellung zu revaskularisierenden Maßnahmen zwischen dem möglichen Nutzen im Sinne einer Verbesserung der linksventrikulären Kontraktionsfunktion und dem deutlich erhöhten Risiko eines operativen oder interventionellen Eingriffs abgewogen werden. Mit nuklearkardiologischen Methoden zur Beurteilung der myokardialen Perfusion, der Zellmembranintegrität und der metabolischen Aktivität in kontraktionsgestörtem Herzmuskelgewebe kann mit hoher diagnostischer Genauigkeit zwischen vitalen und narbigen Myokardregionen differenziert werden. Auch die Mobilisierung der kontraktilen Reserve durch Infusion von Dobutamin bei gleichzeitiger Analyse von regionaler Wandbewegung oder Wanddickenzunahme mittels Echokardiographie oder MRT hat einen hohen diagnostischen Stellenwert. Dies gilt v.a. in Hinblick auf eine korrekte Vorhersage einer $\rightarrow$ linksventrikulären Funktionsverbesserung nach erfolgreicher Revaskularisierung (2). Vielversprechend, da einfach durchführbar und diagnostisch zuverlässig bei allerdings noch geringer Datenbasis, erscheint die MRT-Kontrastmitteluntersuchung zur Differenzierung zwischen Narbe und vitalem Myokard in Verbindung mit einer Beurteilung der Ruhekontraktionsfunktion.

Welche der vorgestellten diagnostischen Strategien zum Vitalitätsnachweis (Perfusion, Zellmembranintegrität/Kontrastmittelanreicherung, metabolische Aktivität oder Nachweis einer dobutamininduzierbaren Kontraktionsreserve) am besten für die Indikationsstellung zu revaskularisierenden Maßnahmen und die Abschätzung der individuellen Prognose geeignet ist, bleibt noch offen. Derzeit hängt die Auswahl der geeigneten Untersuchungsmethode zur Erfassung postischämischer myokardialer Vitalität in 1. Linie von der Verfügbarkeit der Untersuchungstechnik und der Erfahrung des Untersuchers ab. Alle genannten Techniken können bei korrekter Indikationsstellung und Auswertung ca. 80\% der vitalen Myokardareale erfassen. Dabei variiert der positive Vorhersagewert für die Erholung der Kontraktionsfunktion zwischen ca. 60\% und 90\% (Abb.5). Ein Stufenschema zur Vitalitätsdiagnostik ist in Abb. 6 dargestellt. Unabhängig von der verwendeten Untersuchungstechnik ist die Frage der Kosten-Nutzen Relation in der Vitalitätsdiagnostik noch nicht abschließend geklärt. Es ist aber anzunehmen, dass eine zielgerichtete Vitalitätsdiagnostik auch zu einer Kostensenkung im Gesundheitswesen beitragen kann, indem sie bei dem ständig wachsenden Patientenkollektiv mit postischämischer linksventrikulärer Kontraktionsstörung die differentialtherapeutische Entscheidung zwischen Weiterführung einer medikamentösen Therapie oder revaskularisierenden Maßnahmen erleichtert. Die erfolgreiche Revaskularisierung vitaler Myokardareale kann über eine Verbesserung der linksventrikulären Kontraktionsfunktion die Lebensqualität verbessern, die Lebensdauer verlängern und möglicherweise auch die Häufigkeit stationärer Krankenhausbehandlungen und die Notwendigkeit von Herztransplantationen reduzieren, während bei Nachweis von irreversibel geschädigtem Narbengewebe risikoreiche und darüber hinaus teure Revaskularisierungsversuche zugunsten einer konsequenten medikamentösen Therapie unterbleiben können. Erste Studien (CHRISTMAS-Studie) zu medikamentösen Therapiestrategien (z.B. Betablockade) bei Linksherzinsuffizienz unter Einbeziehung des myokardialen Vitalitätsstatus deuten darauf hin, dass dem Vitalitätszustand des Myokards auch eine Bedeutung, z.B. für die Optimierung der medikamentösen Therapie zukommen könnte (5). Ob die Vitalitätsdiagnostik künftig neben der Indikationsstellung zu revaskularisierenden Maßnahmen auch zur Planung einer individuellen medikamentösen Herzinsuffizienztherapie unter Einbeziehung von Vitalitätsparametern führen wird, hängt von den Ergebnissen weiterführender Studien ab.

\section{Fazit}

In der Echokardiographie gilt: Vitales Myokard = positive Kontraktionsreserve: Zunahme der Kontraktilität (Wanddickenzunahme, biphasische Antwort). In der MRT gilt: Vitales Myokard=positive 


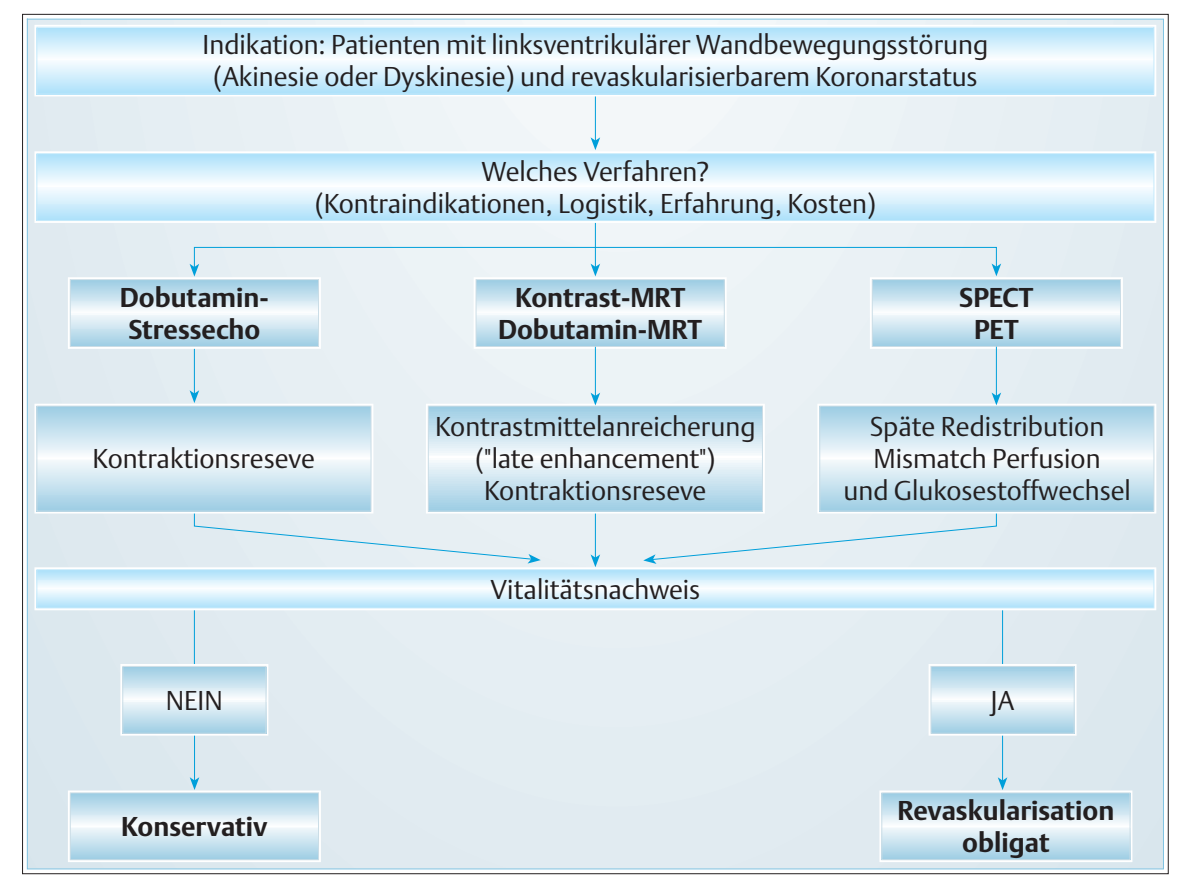

Abb.6 Stufenschema zur Vitalitätsdiagnostik.

Tab.1 Fehlende Kontrastmittelaufnahme (kein „Late enhancement“).

\begin{tabular}{|c|c|}
\hline $\begin{array}{l}\text { Anhaltspunkte für myokardiale } \\
\text { Vitalität }\end{array}$ & \\
\hline Klinik & Postinfarzielle Angina \\
\hline EKG & $\begin{array}{l}\text { Fehlende Q-Zacken, persistierende } \\
\text { R-Zacken }\end{array}$ \\
\hline Koronarangiographie & $\begin{array}{l}\text { Stenose }>70 \% \text {, verzögerte Perfusion, } \\
\text { Kollateralen }\end{array}$ \\
\hline \multicolumn{2}{|l|}{$\begin{array}{l}\text { Weiterführende } \\
\text { Vitalitätsdiagnostik }\end{array}$} \\
\hline Echokardiographie & Dobutamin-Kontraktionsreserve \\
\hline Magnetresonanztomographie & Dobutamin-Kontraktionsreserve \\
\hline Positronenemissionstomographie & Glukose-Metabolismus, Perfusion \\
\hline Myokardszintigraphie & Perfusion, Membranintegrität \\
\hline
\end{tabular}

Kontraktionsreserve: Zunahme der Kontraktilität (Wanddickenzunahme $>2 \mathrm{~mm}$ ). Avitales Myokard=Kontrastmittelaufnahme/Late Enhancement. Eine transmurale Kontrastmittelaufnahme $>50 \%$ der Wand weist auf eine schlechte Prognose $(<5 \%)$ für eine Funktionsverbesserung nach Revaskularisation hin. In der SPECT gilt: Avitales Myokard = Ruheuntersuchung mit Perfusionsdefekt; ggf. auch Reinjektion mit persistierendem Perfusionsdefekt. In der PET gilt: Vitales Myokard: „Mismatch“ zwischen Stoffwechsel und Perfusion, d.h. verminderter Nachweis des Perfusionstracers bei normalem oder gesteigertem Glukose-Stoffwechsel (FDG-Belegung).

Danksagungen: Für Überlassung von Bildmaterial bedanken sich die Autoren herzlich bei Herrn Priv. Doz. Dr. med. D. Moka (Szintigraphie und Positronenemissionstomographie - Klinik und Poliklinik für Nuklearmedizin, Klinikum der Universität zu Köln) und Herrn B. Rückriem (Magnetresonanztomographie - Klinik und Poliklinik für Radiologie, Klinikum der Universität zu Köln).

Autorenerlärung: Die Autoren erklären, dass sie keine finanziellen Verbindungen mit einer Firma haben, deren Produkt in dem Artikel eine wichtige Rolle spielt (oder mit einer Firma, die ein Konkurrenzprodukt vertreibt).

\section{Literatur}

1 Altmann KC, Shaw LJ, Hachamovitch R et al. Myocardial viability testing and impact of revascularization on prognosis in patients with coronary artery disease

2 and left ventricular dysfunction: a meta analysis. JACC 2002; 39: 1151-1158 sesophageal echocardiography and dobutamine-magnetic resonance imaging for the prediction of left ventricular functional recovery in patients with chronic

3 Coronary artery disease. Eur Heart J 2000; 21: 981-991 nal and morphologic approach to the detection of residual myocardial viability.

4 Circulation 1995; 91: 1006-1015 ventricular dysfunction. Circulation 1982; 66: 1146-1149

5 ventricular dysfunction. Circulation 1982; 66: 1146-1149 Cleland JGF, Pennell DJ, Ray SG et al. Myocardial viability as a determinant of the
ejection fraction response to carvedilol in patients with heart failure (CHRISTejection fraction response to carvedilo
MAS trial). Lancet 2003; 362: 14-21

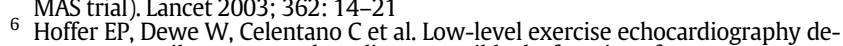
tects contractile reserve and predicts reversible dysfunction after acute myocardial infarction. J Am Coll Cardiol 1999; 34: 989-997

Kim JR, Fieno DS, Parrish TB et al. Relationship of MRI delayed contrast enhancement to irreversible injury, infarct age, and contractile function. Circulation 1999; 100: 1992-2002

Knuesel RP, Nanz D, Wyss C et al. Characterization of Dysfunctional Myocardium by Positron Emission Tomography and Magnetic Resonance: Relation to Functional Outcome After Revascularization. Circulation 2003; 108: 1095-1100

9 Mahrholdt H, Wagner A, Holly TA et al. Reproducibility of chronic infarct size measurement by contrast-enhanced magnetic resonance imaging. Circulation 2002; 106: 2322-2327

10 Nesto RW, Cohn LH, Collins JJ et al. Inotropic contractile reserve: a useful predictor of increased 5-year survival and improved postoperative left ventricular function in patients with coronary artery disease and reduced ejection fraction. Am I Cardiol 1982; 50: 39-44

11 Nixdorff U. Vitalitätsdiagnostik. Thieme 2002, In: Flachskampf FA, Hrsg.. Praxis der Echokardiographie. 2002: 204-216

12 Rahimtoola SH. The hibernating myocardium. Am Heart J 1989; 117: 211-221

13 Rasmussen S, Corya B, Feigenbaum H, Knoebel S. Detection of myocardial scar

4 tissue by M-mode echocardiography. Circulation 1978; 57: 230-238 ischemic cardiomyopathy. Echocardiography 2000; $27: 69-77$

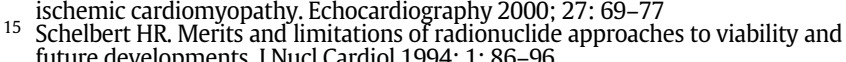

16 future developments. J Nucl Cardiol 1994; 1: 86-96 Tillisch J, Brunken R, Marshall R et al. Reversibility of cardiac wall motion abno

17 malities predicted by positron tomography. N Eng J Med 1986; 314: 884-888 Vom Dahl J, Altehoefer C, Sheehan $\mathrm{H}$ et al. Effect of myocardial viability assessed by technetium-99m-sestamibi SPECT and Fluorine-18-FDG PET on clinical out-

18 come in coronary artery disease. J Nucl Med 1997; 38: 742-748

Kim R, Wu E, Rafael A et al. The use of contrast-enhanced magnetic resonance imaging to identify reversible myocardial dysfunction. N Eng J Med 2000; 343 : 1445-1453 


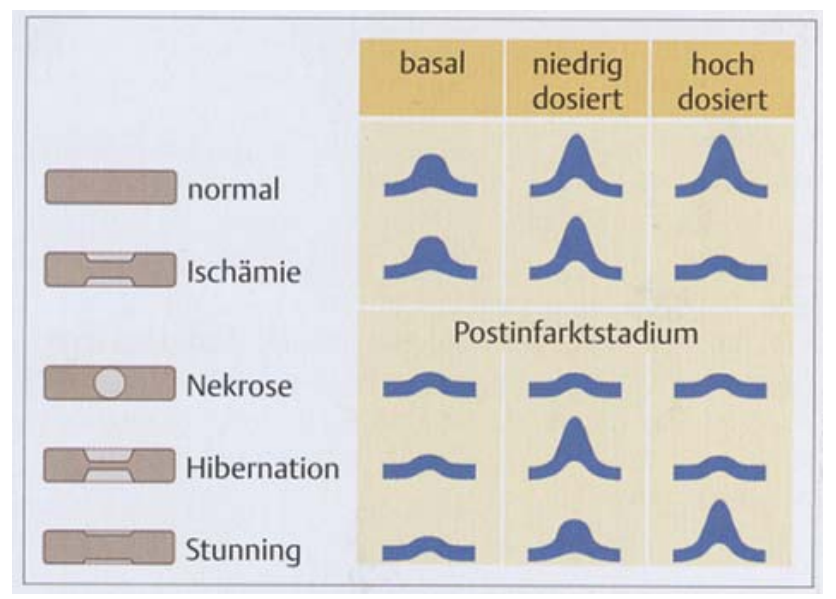

Abb.4 Schematische Darstellung der verschiedenen Reaktionsweisen des Myokards auf eine sequenzielle Dobutamintitration (DobutaminEchokardiographie). Die linke Spalte repräsentiert die koronare Pathomorphologie, die rechte Spalte die Wandbewegung und -verdickung während niedrig und hoch dosierter Dobutamintitration. Die verschiedenen Zeilen demonstrieren die normale, ischämische und Postinfarktsituation (Quelle: Flachskampf FA (Hrsg.), Praxis der Echokardiographie. Thieme; 2002: 204-216) 